Acta Crystallographica Section E

Structure Reports

Online

ISSN 1600-5368

\section{1-[(Pyridin-3-yl)(pyrrolidin-1-yl)methyl]- naphthalen-2-ol}

\section{Qin-Qin Zhou}

Ordered Matter Science Research Center, College of Chemistry and Chemical, Engineering, Southeast University, Nanjing 211189, People's Republic of China Correspondence e-mail: zhouqinqin623@sina.com

Received 31 March 2012; accepted 24 July 2012

Key indicators: single-crystal X-ray study; $T=293 \mathrm{~K}$; mean $\sigma(\mathrm{C}-\mathrm{C})=0.005 \AA$; $R$ factor $=0.050 ; w R$ factor $=0.122 ;$ data-to-parameter ratio $=8.9$.

The title compound, $\mathrm{C}_{20} \mathrm{H}_{20} \mathrm{~N}_{2} \mathrm{O}$, was synthesized by a solventfree one-pot three-component domino reaction of naphthalen-2-ol, nicotinaldehyde and pyrrolidine. The dihedral angle between the naphthalene ring system and the pyridine ring is $74.22(6)^{\circ}$. The pyrrolidine ring assumes an envelope conformation with the $\mathrm{N}$ atom as the flap. An intramolecular $\mathrm{O}-$ $\mathrm{H} \cdots \mathrm{N}$ hydrogen bond stabilizes the molecular conformation.

\section{Related literature}

For the synthesis and structure of a related compound, see: Wang (2012).

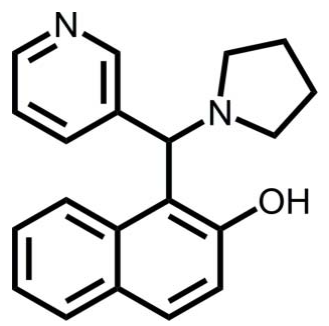

\section{Experimental}

Crystal data

$\mathrm{C}_{20} \mathrm{H}_{20} \mathrm{~N}_{2} \mathrm{O}$

$M_{r}=304.38$
Monoclinic, $C c$

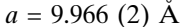

$b=15.587$ (3) $\AA$

$c=10.477(2) \AA$

$\beta=91.60(3)^{\circ}$

$V=1626.9(6) \AA^{3}$

$Z=4$

Mo $K \alpha$ radiation

$\mu=0.08 \mathrm{~mm}^{-1}$

$T=293 \mathrm{~K}$

$0.38 \times 0.32 \times 0.27 \mathrm{~mm}$

Data collection

Rigaku SCXmini CCD diffractometer

Absorption correction: multi-scan (CrystalClear; Rigaku, 2005)

$T_{\min }=0.967, T_{\max }=0.982$

Refinement

$R\left[F^{2}>2 \sigma\left(F^{2}\right)\right]=0.050$

$w R\left(F^{2}\right)=0.122$

$S=1.02$

1857 reflections

209 parameters

8194 measured reflections 1857 independent reflections 1361 reflections with $I>2 \sigma(I)$ $R_{\text {int }}=0.067$

Table 1

Hydrogen-bond geometry $\left(\AA{ }^{\circ}\right)$.

\begin{tabular}{lllll}
\hline$D-\mathrm{H} \cdots A$ & $D-\mathrm{H}$ & $\mathrm{H} \cdots A$ & $D \cdots A$ & $D-\mathrm{H} \cdots A$ \\
\hline $\mathrm{O} 1-\mathrm{H} 1 \cdots \mathrm{N} 1$ & 0.82 & 1.85 & $2.572(3)$ & 147
\end{tabular}

Data collection: CrystalClear (Rigaku,2005); cell refinement: CrystalClear; data reduction: CrystalClear; program(s) used to solve structure: SHELXS97 (Sheldrick, 2008); program(s) used to refine structure: SHELXL97 (Sheldrick, 2008); molecular graphics: DIAMOND (Brandenburg \& Putz, 2005); software used to prepare material for publication: SHELXL97.

This work was supported by Southeast University

Supplementary data and figures for this paper are available from the IUCr electronic archives (Reference: MW2063).

\section{References}

Brandenburg, K. \& Putz, H. (2005). DIAMOND. Crystal Impact GbR, Bonn, Germany.

Rigaku (2005). CrystalClear. Rigaku Corporation, Tokyo, Japan.

Sheldrick, G. M. (2008). Acta Cryst. A64, 112-122.

Wang, W. (2012). Acta Cryst. E68, o884. 


\section{supporting information}

Acta Cryst. (2012). E68, o2614 [doi:10.1107/S1600536812033363]

\section{1-[(Pyridin-3-yl)(pyrrolidin-1-yl)methyl]naphthalen-2-ol}

\section{Qin-Qin Zhou}

\section{S1. Comment}

The so-called Betti base derivatives, which can be synthesized by many routes (Wang, 2012), have been of great interest in coordination chemistry. Herein the crystal structure of one such compound, obtained by a solvent-free, one-pot, threecomponent, domino reaction of naphthalen-2-ol, nicotinaldehyde and pyrrolidine is reported.

In the title compound the bond lengths and angles are well within the expected ranges. The dihedral angle between the naphthalene ring system and the pyridine ring is $74.22(6)^{\circ}$. The pyrrolidine ring adopts an envelope conformation. An intramolecular $\mathrm{O}-\mathrm{H} \cdots \mathrm{N}$ hydrogen bond (Table 1) stabilizes the molecular conformation.

\section{S2. Experimental}

A dry $50 \mathrm{~mL}$ flask was charged with nicotinaldehyde $(10 \mathrm{mmol})$, naphthalen-2-ol $(10 \mathrm{mmol})$ and pyrrolidine $(10 \mathrm{mmol})$. The mixture was stirred at $100^{\circ} \mathrm{C}$ for $5 \mathrm{~h}$ and then ethanol $(15 \mathrm{~mL})$ was added. After refluxing for 30 minutes, the solution was filtered and crystals of the title compound suitable for X-ray analysis were obtained by slow evaporation.

\section{S3. Refinement}

All $\mathrm{H}$ atoms were calculated geometrically and refined using a riding model with $\mathrm{C}-\mathrm{H}=0.93-0.98 \AA, \mathrm{O}-\mathrm{H}=0.82 \AA$ and with $U_{\text {iso }}(\mathrm{H})=1.2 U_{\text {eq }}(\mathrm{C})$ for carbon-bound or $1.5 U_{\text {eq }}(\mathrm{O})$ for oxygen-bound $\mathrm{H}$ atoms.
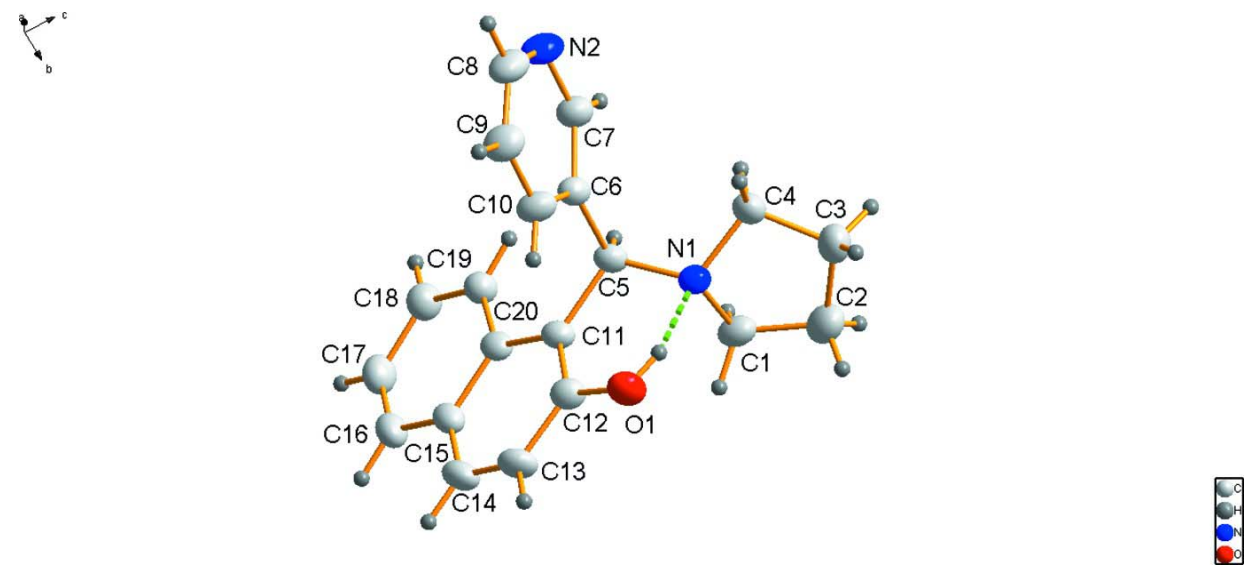

\section{Figure 1}

The structure of the title compound with displacement ellipsoids drawn at the $30 \%$ probability level. The intramolecular hydrogen bond is shown as a dashed line. 


\section{1-[(Pyridin-3-yl)(pyrrolidin-1-yl)methyl]naphthalen-2-ol}

Crystal data

$$
\begin{aligned}
& \mathrm{C}_{20} \mathrm{H}_{20} \mathrm{~N}_{2} \mathrm{O} \\
& M_{r}=304.38 \\
& \text { Monoclinic, } C c \\
& \text { Hall symbol: } \mathrm{C}-2 \mathrm{yc} \\
& a=9.966(2) \AA \\
& b=15.587(3) \AA \\
& c=10.477(2) \AA \\
& \beta=91.60(3)^{\circ} \\
& V=1626.9(6) \AA^{3} \\
& Z=4
\end{aligned}
$$

\section{Data collection}

Rigaku SCXmini CCD diffractometer

Radiation source: fine-focus sealed tube Graphite monochromator $\omega$ scans

Absorption correction: multi-scan

(CrystalClear; Rigaku, 2005)

$T_{\min }=0.967, T_{\max }=0.982$

\section{Refinement}

Refinement on $F^{2}$

Least-squares matrix: full

$R\left[F^{2}>2 \sigma\left(F^{2}\right)\right]=0.050$

$w R\left(F^{2}\right)=0.122$

$S=1.02$

1857 reflections

209 parameters

2 restraints

Primary atom site location: structure-invariant direct methods
$F(000)=648$

$D_{\mathrm{x}}=1.243 \mathrm{Mg} \mathrm{m}^{-3}$

Mo $K \alpha$ radiation, $\lambda=0.71073 \AA$

Cell parameters from 3664 reflections

$\theta=3.1-27.5^{\circ}$

$\mu=0.08 \mathrm{~mm}^{-1}$

$T=293 \mathrm{~K}$

Block, colourless

$0.38 \times 0.32 \times 0.27 \mathrm{~mm}$

8194 measured reflections

1857 independent reflections

1361 reflections with $I>2 \sigma(I)$

$R_{\text {int }}=0.067$

$\theta_{\max }=27.5^{\circ}, \theta_{\min }=3.1^{\circ}$

$h=-12 \rightarrow 12$

$k=-20 \rightarrow 20$

$l=-13 \rightarrow 13$

Secondary atom site location: difference Fourier map

Hydrogen site location: inferred from neighbouring sites

$\mathrm{H}$-atom parameters constrained

$w=1 /\left[\sigma^{2}\left(F_{\mathrm{o}}^{2}\right)+(0.0577 P)^{2}\right]$

where $P=\left(F_{\mathrm{o}}^{2}+2 F_{\mathrm{c}}^{2}\right) / 3$

$(\Delta / \sigma)_{\max }<0.001$

$\Delta \rho_{\max }=0.13$ e $\AA^{-3}$

$\Delta \rho_{\min }=-0.13$ e $\AA^{-3}$

\section{Special details}

Geometry. All e.s.d.'s (except the e.s.d. in the dihedral angle between two 1.s. planes) are estimated using the full covariance matrix. The cell e.s.d.'s are taken into account individually in the estimation of e.s.d.'s in distances, angles and torsion angles; correlations between e.s.d.'s in cell parameters are only used when they are defined by crystal symmetry. An approximate (isotropic) treatment of cell e.s.d.'s is used for estimating e.s.d.'s involving 1.s. planes.

Refinement. Refinement of $F^{2}$ against ALL reflections. The weighted $R$-factor $w R$ and goodness of fit $S$ are based on $F^{2}$, conventional $R$-factors $R$ are based on $F$, with $F$ set to zero for negative $F^{2}$. The threshold expression of $F^{2}>\sigma\left(F^{2}\right)$ is used only for calculating $R$-factors(gt) etc. and is not relevant to the choice of reflections for refinement. $R$-factors based on $F^{2}$ are statistically about twice as large as those based on $F$, and $R$ - factors based on ALL data will be even larger.

Fractional atomic coordinates and isotropic or equivalent isotropic displacement parameters $\left(\hat{A}^{2}\right)$

\begin{tabular}{lllll}
\hline & $x$ & $y$ & $z$ & $U_{\text {iso }} * / U_{\text {eq }}$ \\
\hline C1 & $-0.0405(4)$ & $0.2124(2)$ & $0.2764(4)$ & $0.0609(9)$ \\
H1A & -0.1100 & 0.1705 & 0.2925 & $0.073^{*}$ \\
H1B & -0.0724 & 0.2515 & 0.2102 & $0.073^{*}$ \\
C2 & $-0.0017(5)$ & $0.2605(3)$ & $0.3966(4)$ & $0.0862(13)$
\end{tabular}




$\begin{array}{lllll}\text { H2A } & -0.0749 & 0.2599 & 0.4557 & 0.103^{*} \\ \text { H2B } & 0.0199 & 0.3196 & 0.3770 & 0.103^{*} \\ \text { C3 } & 0.1203(4) & 0.2148(3) & 0.4541(3) & 0.0728(1) \\ \text { H3A } & 0.1014 & 0.1924 & 0.5381 & 0.087^{*} \\ \text { H3B } & 0.1964 & 0.2534 & 0.4614 & 0.087^{*} \\ \text { C4 } & 0.1484(4) & 0.1422(2) & 0.3617(3) & 0.0560(8) \\ \text { H4A } & 0.2443 & 0.1341 & 0.3534 & 0.067^{*} \\ \text { H4B } & 0.1091 & 0.0890 & 0.3907 & 0.067^{*} \\ \text { C5 } & 0.0588(3) & 0.09834(19) & 0.1473(3) & 0.0452(7) \\ \text { H5 } & -0.0119 & 0.0622 & 0.1820 & 0.054^{*} \\ \text { C6 } & 0.1826(3) & 0.04266(18) & 0.1305(3) & 0.0447(7) \\ \text { C7 } & 0.1969(3) & -0.0334(2) & 0.1964(3) & 0.0575(8) \\ \text { H7 } & 0.1296 & -0.0480 & 0.2522 & 0.069^{*} \\ \text { C8 } & 0.3946(4) & -0.0638(2) & 0.1086(4) & 0.0711(10) \\ \text { H8 } & 0.4690 & -0.0994 & 0.1020 & 0.085^{*} \\ \text { C9 } & 0.3914(4) & 0.0095(2) & 0.0368(4) & 0.0701(10) \\ \text { H9 } & 0.4606 & 0.0225 & -0.0178 & 0.084^{*} \\ \text { C10 } & 0.2826(3) & 0.0636(2) & 0.0475(4) & 0.0587(9) \\ \text { H10 } & 0.2768 & 0.1137 & -0.0007 & 0.070^{*} \\ \text { C11 } & 0.0069(3) & 0.13455(19) & 0.0201(3) & 0.0457(7) \\ \text { C12 } & 0.0554(3) & 0.2112(2) & -0.0248(3) & 0.0521(8) \\ \text { C13 } & 0.0112(3) & 0.2441(2) & -0.1444(3) & 0.0596(9) \\ \text { H13 } & 0.0478 & 0.2949 & -0.1744 & 0.072^{*} \\ \text { C14 } & -0.0833(4) & 0.2028(2) & -0.2156(3) & 0.0626(10) \\ \text { H14 } & -0.1114 & 0.2256 & -0.2939 & 0.075^{*} \\ \text { C15 } & -0.1403(3) & 0.1251(2) & -0.1726(3) & 0.0528(8) \\ \text { C16 } & -0.2440(4) & 0.0822(3) & -0.2422(3) & 0.0635(10) \\ \text { H16 } & -0.2781 & 0.1066 & -0.3174 & 0.076^{*} \\ \text { C17 } & -0.2945(4) & 0.0070(3) & -0.2023(4) & 0.0688(10) \\ \text { H17 } & -0.3624 & -0.0202 & -0.2498 & 0.083^{*} \\ \text { C18 } & -0.2443(3) & -0.0301(2) & -0.0888(3) & 0.0614(9) \\ \text { H18 } & -0.2789 & -0.0822 & -0.0614 & 0.074^{*} \\ \text { C19 } & -0.1451(3) & 0.0095(2) & -0.0180(3) & 0.0526(8) \\ \text { H19 } & -0.1118 & -0.0168 & 0.0560 & 0.063^{*} \\ \text { C20 } & -0.0921(3) & 0.08934(19) & -0.0545(3) & 0.0465(7) \\ \text { N1 } & 0.0857(3) & 0.16953(15) & 0.2391(2) & 0.0476(6) \\ \text { N2 } & 0.2995(3) & -0.08781(18) & 0.1867(3) & 0.0720(9) \\ \text { O1 } & 0.1471(2) & 0.25894(14) & 0.0428(2) & 0.0616(6) \\ \text { H1 } & 0.1554 & 0.2398 & 0.1155 & 0.092^{*} \\ & & & & \end{array}$

Atomic displacement parameters $\left(\AA^{2}\right)$

\begin{tabular}{lllllll}
\hline & $U^{11}$ & $U^{22}$ & $U^{33}$ & $U^{12}$ & $U^{13}$ & $U^{23}$ \\
\hline C1 & $0.061(2)$ & $0.0530(18)$ & $0.070(2)$ & $0.0123(16)$ & $0.0124(17)$ & $0.0068(17)$ \\
C2 & $0.105(4)$ & $0.079(3)$ & $0.076(3)$ & $0.026(3)$ & $0.019(3)$ & $-0.005(2)$ \\
C3 & $0.091(3)$ & $0.072(2)$ & $0.055(2)$ & $0.002(2)$ & $0.007(2)$ & $-0.0058(18)$ \\
C4 & $0.065(2)$ & $0.0566(19)$ & $0.0461(17)$ & $0.0057(16)$ & $-0.0002(15)$ & $0.0067(15)$ \\
C5 & $0.0402(15)$ & $0.0456(18)$ & $0.0496(16)$ & $-0.0014(13)$ & $-0.0030(13)$ & $0.0079(13)$
\end{tabular}


supporting information

$\begin{array}{lllllll}\text { C6 } & 0.0420(16) & 0.0414(15) & 0.0502(16) & -0.0009(13) & -0.0074(13) & 0.0022(13) \\ \text { C7 } & 0.055(2) & 0.0502(18) & 0.067(2) & 0.0037(15) & -0.0013(16) & 0.0044(16) \\ \text { C8 } & 0.057(2) & 0.062(2) & 0.094(3) & 0.0183(18) & -0.012(2) & -0.006(2) \\ \text { C9 } & 0.051(2) & 0.073(2) & 0.087(3) & 0.0090(17) & 0.0106(19) & -0.001(2) \\ \text { C10 } & 0.0516(19) & 0.0483(18) & 0.076(2) & 0.0023(15) & 0.0050(18) & 0.0067(17) \\ \text { C11 } & 0.0413(16) & 0.0467(18) & 0.0490(17) & 0.0039(13) & -0.0009(14) & 0.0079(13) \\ \text { C12 } & 0.0474(18) & 0.0503(19) & 0.059(2) & 0.0009(14) & 0.0017(16) & 0.0061(15) \\ \text { C13 } & 0.065(2) & 0.056(2) & 0.058(2) & 0.0037(17) & 0.0039(18) & 0.0202(16) \\ \text { C14 } & 0.068(2) & 0.070(2) & 0.0501(19) & 0.0148(18) & -0.0006(18) & 0.0165(17) \\ \text { C15 } & 0.0473(17) & 0.0655(19) & 0.0453(17) & 0.0141(16) & -0.0021(14) & 0.0004(15) \\ \text { C16 } & 0.061(2) & 0.078(3) & 0.0500(19) & 0.0149(19) & -0.0091(18) & -0.0046(18) \\ \text { C17 } & 0.062(2) & 0.080(3) & 0.063(2) & 0.001(2) & -0.0129(18) & -0.016(2) \\ \text { C18 } & 0.054(2) & 0.068(2) & 0.062(2) & -0.0062(16) & -0.0013(18) & -0.0053(18) \\ \text { C19 } & 0.0503(18) & 0.0544(18) & 0.0529(18) & 0.0018(14) & -0.0016(15) & 0.0019(15) \\ \text { C20 } & 0.0382(15) & 0.0534(17) & 0.0479(16) & 0.0102(13) & 0.0027(13) & 0.0026(13) \\ \text { N1 } & 0.0490(14) & 0.0443(13) & 0.0497(14) & 0.0044(11) & 0.0034(12) & 0.0036(11) \\ \text { N2 } & 0.067(2) & 0.0563(19) & 0.092(2) & 0.0165(16) & -0.0065(19) & 0.0065(17) \\ \text { O1 } & 0.0666(15) & 0.0538(13) & 0.0643(14) & -0.0110(12) & -0.0020(13) & 0.0119(11) \\ & & & & & & \end{array}$

Geometric parameters $\left(\AA,{ }^{\circ}\right)$

\begin{tabular}{llll}
\hline $\mathrm{C} 1-\mathrm{N} 1$ & $1.486(4)$ & $\mathrm{C} 8-\mathrm{H} 8$ & 0.9300 \\
$\mathrm{C} 1-\mathrm{C} 2$ & $1.506(6)$ & $\mathrm{C} 9-\mathrm{C} 10$ & $1.381(5)$ \\
$\mathrm{C} 1-\mathrm{H} 1 \mathrm{~A}$ & 0.9700 & $\mathrm{C} 9-\mathrm{H} 9$ & 0.9300 \\
$\mathrm{C} 1-\mathrm{H} 1 \mathrm{~B}$ & 0.9700 & $\mathrm{C} 10-\mathrm{H} 10$ & 0.9300 \\
$\mathrm{C} 2-\mathrm{C} 3$ & $1.520(6)$ & $\mathrm{C} 11-\mathrm{C} 12$ & $1.376(4)$ \\
$\mathrm{C} 2-\mathrm{H} 2 \mathrm{~A}$ & 0.9700 & $\mathrm{C} 11-\mathrm{C} 20$ & $1.427(4)$ \\
$\mathrm{C} 2-\mathrm{H} 2 \mathrm{~B}$ & 0.9700 & $\mathrm{C} 12-\mathrm{O} 1$ & $1.362(4)$ \\
$\mathrm{C} 3-\mathrm{C} 4$ & $1.520(5)$ & $\mathrm{C} 12-\mathrm{C} 13$ & $1.413(4)$ \\
$\mathrm{C} 3-\mathrm{H} 3 \mathrm{~A}$ & 0.9700 & $\mathrm{C} 13-\mathrm{C} 14$ & $1.349(5)$ \\
$\mathrm{C} 3-\mathrm{H} 3 \mathrm{~B}$ & 0.9700 & $\mathrm{C} 13-\mathrm{H} 13$ & 0.9300 \\
$\mathrm{C} 4-\mathrm{N} 1$ & $1.476(4)$ & $\mathrm{C} 14-\mathrm{C} 15$ & $1.416(5)$ \\
$\mathrm{C} 4-\mathrm{H} 4 \mathrm{~A}$ & 0.9700 & $\mathrm{C} 14-\mathrm{H} 14$ & 0.9300 \\
$\mathrm{C} 4-\mathrm{H} 4 \mathrm{~B}$ & 0.9700 & $\mathrm{C} 15-\mathrm{C} 16$ & $1.416(5)$ \\
$\mathrm{C} 5-\mathrm{N} 1$ & $1.488(4)$ & $\mathrm{C} 15-\mathrm{C} 20$ & $1.428(4)$ \\
$\mathrm{C} 5-\mathrm{C} 6$ & $1.523(4)$ & $\mathrm{C} 16-\mathrm{C} 17$ & $1.347(5)$ \\
$\mathrm{C} 5-\mathrm{C} 11$ & $1.524(4)$ & $\mathrm{C} 16-\mathrm{H} 16$ & 0.9300 \\
$\mathrm{C} 5-\mathrm{H} 5$ & 0.9800 & $\mathrm{C} 17-\mathrm{C} 18$ & $1.402(5)$ \\
$\mathrm{C} 6-\mathrm{C} 7$ & $1.377(4)$ & $\mathrm{C} 17-\mathrm{H} 17$ & 0.9300 \\
$\mathrm{C} 6-\mathrm{C} 10$ & $1.380(4)$ & $\mathrm{C} 18-\mathrm{C} 19$ & $1.367(5)$ \\
$\mathrm{C} 7-\mathrm{N} 2$ & $1.334(4)$ & $\mathrm{C} 18-\mathrm{H} 18$ & 0.9300 \\
$\mathrm{C} 7-\mathrm{H} 7$ & $\mathrm{C} 19-\mathrm{C} 20$ & $1.409(4)$ \\
$\mathrm{C} 8-\mathrm{N} 2$ & 1.9300 & $\mathrm{C} 19-\mathrm{H} 19$ & 0.9300 \\
$\mathrm{C} 8-\mathrm{C} 9$ & $\mathrm{O} 1-\mathrm{H} 1$ & 0.8200 \\
& $1.368(6)$ & $\mathrm{C} 8-\mathrm{C} 9-\mathrm{H} 9$ & 120.9 \\
$\mathrm{~N} 1-\mathrm{C} 1-\mathrm{C} 2$ & & $\mathrm{C} 10-\mathrm{C} 9-\mathrm{H} 9$ & 120.9 \\
$\mathrm{~N} 1-\mathrm{C} 1-\mathrm{H} 1 \mathrm{~A}$ & $104.2(3)$ & $\mathrm{C} 6-\mathrm{C} 10-\mathrm{C} 9$ & $119.3(3)$ \\
$\mathrm{C} 2-\mathrm{C} 1-\mathrm{H} 1 \mathrm{~A}$ & 110.9 & &
\end{tabular}




\begin{tabular}{|c|c|c|c|}
\hline $\mathrm{N} 1-\mathrm{C} 1-\mathrm{H} 1 \mathrm{~B}$ & 110.9 & $\mathrm{C} 6-\mathrm{C} 10-\mathrm{H} 10$ & 120.4 \\
\hline $\mathrm{C} 2-\mathrm{C} 1-\mathrm{H} 1 \mathrm{~B}$ & 110.9 & $\mathrm{C} 9-\mathrm{C} 10-\mathrm{H} 10$ & 120.4 \\
\hline $\mathrm{H} 1 \mathrm{~A}-\mathrm{C} 1-\mathrm{H} 1 \mathrm{~B}$ & 108.9 & $\mathrm{C} 12-\mathrm{C} 11-\mathrm{C} 20$ & $119.0(3)$ \\
\hline $\mathrm{C} 1-\mathrm{C} 2-\mathrm{C} 3$ & $106.4(3)$ & $\mathrm{C} 12-\mathrm{C} 11-\mathrm{C} 5$ & $120.4(3)$ \\
\hline $\mathrm{C} 1-\mathrm{C} 2-\mathrm{H} 2 \mathrm{~A}$ & 110.5 & $\mathrm{C} 20-\mathrm{C} 11-\mathrm{C} 5$ & $120.6(2)$ \\
\hline $\mathrm{C} 3-\mathrm{C} 2-\mathrm{H} 2 \mathrm{~A}$ & 110.5 & $\mathrm{O} 1-\mathrm{C} 12-\mathrm{C} 11$ & $122.2(3)$ \\
\hline $\mathrm{C} 1-\mathrm{C} 2-\mathrm{H} 2 \mathrm{~B}$ & 110.5 & $\mathrm{O} 1-\mathrm{C} 12-\mathrm{C} 13$ & $116.8(3)$ \\
\hline $\mathrm{C} 3-\mathrm{C} 2-\mathrm{H} 2 \mathrm{~B}$ & 110.5 & $\mathrm{C} 11-\mathrm{C} 12-\mathrm{C} 13$ & $121.0(3)$ \\
\hline $\mathrm{H} 2 \mathrm{~A}-\mathrm{C} 2-\mathrm{H} 2 \mathrm{~B}$ & 108.6 & $\mathrm{C} 14-\mathrm{C} 13-\mathrm{C} 12$ & $120.8(3)$ \\
\hline $\mathrm{C} 2-\mathrm{C} 3-\mathrm{C} 4$ & $104.8(3)$ & $\mathrm{C} 14-\mathrm{C} 13-\mathrm{H} 13$ & 119.6 \\
\hline $\mathrm{C} 2-\mathrm{C} 3-\mathrm{H} 3 \mathrm{~A}$ & 110.8 & $\mathrm{C} 12-\mathrm{C} 13-\mathrm{H} 13$ & 119.6 \\
\hline $\mathrm{C} 4-\mathrm{C} 3-\mathrm{H} 3 \mathrm{~A}$ & 110.8 & $\mathrm{C} 13-\mathrm{C} 14-\mathrm{C} 15$ & $120.8(3)$ \\
\hline $\mathrm{C} 2-\mathrm{C} 3-\mathrm{H} 3 \mathrm{~B}$ & 110.8 & $\mathrm{C} 13-\mathrm{C} 14-\mathrm{H} 14$ & 119.6 \\
\hline $\mathrm{C} 4-\mathrm{C} 3-\mathrm{H} 3 \mathrm{~B}$ & 110.8 & $\mathrm{C} 15-\mathrm{C} 14-\mathrm{H} 14$ & 119.6 \\
\hline $\mathrm{H} 3 \mathrm{~A}-\mathrm{C} 3-\mathrm{H} 3 \mathrm{~B}$ & 108.9 & $\mathrm{C} 16-\mathrm{C} 15-\mathrm{C} 14$ & $122.3(3)$ \\
\hline $\mathrm{N} 1-\mathrm{C} 4-\mathrm{C} 3$ & 105.0 & $\mathrm{C} 16-\mathrm{C} 15-\mathrm{C} 20$ & $119.0(3)$ \\
\hline $\mathrm{N} 1-\mathrm{C} 4-\mathrm{H} 4 \mathrm{~A}$ & 110.7 & $\mathrm{C} 14-\mathrm{C} 15-\mathrm{C} 20$ & $118.8(3)$ \\
\hline $\mathrm{C} 3-\mathrm{C} 4-\mathrm{H} 4 \mathrm{~A}$ & 110.7 & $\mathrm{C} 17-\mathrm{C} 16-\mathrm{C} 15$ & $121.7(3)$ \\
\hline $\mathrm{N} 1-\mathrm{C} 4-\mathrm{H} 4 \mathrm{~B}$ & 110.7 & $\mathrm{C} 17-\mathrm{C} 16-\mathrm{H} 16$ & 119.1 \\
\hline $\mathrm{C} 3-\mathrm{C} 4-\mathrm{H} 4 \mathrm{~B}$ & 110.7 & $\mathrm{C} 15-\mathrm{C} 16-\mathrm{H} 16$ & 119.2 \\
\hline $\mathrm{H} 4 \mathrm{~A}-\mathrm{C} 4-\mathrm{H} 4 \mathrm{~B}$ & 108.8 & $\mathrm{C} 16-\mathrm{C} 17-\mathrm{C} 18$ & $119.6(3)$ \\
\hline $\mathrm{N} 1-\mathrm{C} 5-\mathrm{C} 6$ & $111.6(2)$ & $\mathrm{C} 16-\mathrm{C} 17-\mathrm{H} 17$ & 120.2 \\
\hline $\mathrm{N} 1-\mathrm{C} 5-\mathrm{C} 11$ & $109.8(2)$ & $\mathrm{C} 18-\mathrm{C} 17-\mathrm{H} 17$ & 120.2 \\
\hline $\mathrm{C} 6-\mathrm{C} 5-\mathrm{C} 11$ & $111.5(2)$ & $\mathrm{C} 19-\mathrm{C} 18-\mathrm{C} 17$ & $120.6(4)$ \\
\hline $\mathrm{N} 1-\mathrm{C} 5-\mathrm{H} 5$ & 107.9 & $\mathrm{C} 19-\mathrm{C} 18-\mathrm{H} 18$ & 119.7 \\
\hline $\mathrm{C} 6-\mathrm{C} 5-\mathrm{H} 5$ & 107.9 & $\mathrm{C} 17-\mathrm{C} 18-\mathrm{H} 18$ & 119.7 \\
\hline $\mathrm{C} 11-\mathrm{C} 5-\mathrm{H} 5$ & 107.9 & $\mathrm{C} 18-\mathrm{C} 19-\mathrm{C} 20$ & $121.5(3)$ \\
\hline $\mathrm{C} 7-\mathrm{C} 6-\mathrm{C} 10$ & $116.9(3)$ & $\mathrm{C} 18-\mathrm{C} 19-\mathrm{H} 19$ & 119.2 \\
\hline $\mathrm{C} 7-\mathrm{C} 6-\mathrm{C} 5$ & $120.4(3)$ & $\mathrm{C} 20-\mathrm{C} 19-\mathrm{H} 19$ & 119.2 \\
\hline $\mathrm{C} 10-\mathrm{C} 6-\mathrm{C} 5$ & $122.6(3)$ & $\mathrm{C} 19-\mathrm{C} 20-\mathrm{C} 11$ & $123.1(3)$ \\
\hline $\mathrm{N} 2-\mathrm{C} 7-\mathrm{C} 6$ & $125.3(3)$ & $\mathrm{C} 19-\mathrm{C} 20-\mathrm{C} 15$ & $117.4(3)$ \\
\hline $\mathrm{N} 2-\mathrm{C} 7-\mathrm{H} 7$ & 117.3 & $\mathrm{C} 11-\mathrm{C} 20-\mathrm{C} 15$ & $119.5(3)$ \\
\hline $\mathrm{C} 6-\mathrm{C} 7-\mathrm{H} 7$ & 117.3 & $\mathrm{C} 4-\mathrm{N} 1-\mathrm{C} 1$ & $104.0(2)$ \\
\hline $\mathrm{N} 2-\mathrm{C} 8-\mathrm{C} 9$ & $124.8(3)$ & $\mathrm{C} 4-\mathrm{N} 1-\mathrm{C} 5$ & $114.3(2)$ \\
\hline $\mathrm{N} 2-\mathrm{C} 8-\mathrm{H} 8$ & 117.6 & $\mathrm{C} 1-\mathrm{N} 1-\mathrm{C} 5$ & $111.5(3)$ \\
\hline $\mathrm{C} 9-\mathrm{C} 8-\mathrm{H} 8$ & 117.6 & $\mathrm{C} 8-\mathrm{N} 2-\mathrm{C} 7$ & $115.5(3)$ \\
\hline $\mathrm{C} 8-\mathrm{C} 9-\mathrm{C} 10$ & $118.1(4)$ & $\mathrm{C} 12-\mathrm{O} 1-\mathrm{H} 1$ & 109.5 \\
\hline
\end{tabular}

Hydrogen-bond geometry $\left(\AA,{ }^{\circ}\right)$

\begin{tabular}{lllll}
\hline$D-\mathrm{H} \cdots A$ & $D-\mathrm{H}$ & $\mathrm{H} \cdots A$ & $D \cdots A$ & $D-\mathrm{H} \cdots A$ \\
\hline $\mathrm{O} 1-\mathrm{H} 1 \cdots \mathrm{N} 1$ & 0.82 & 1.85 & $2.572(3)$ & 147 \\
\hline
\end{tabular}

